\title{
Model Study for Early Warning System of Urban Road Intersection Based on the Back Propagation Neural Network
}

\author{
Wang Qiquan, Fei Yuzhou, Ni Junwen \\ Safety Engineering, China Institute of Industrial Relations, Beijing, China
}

Email address:

wqq_100@163.com (Wang Qiquan)

To cite this article:

Wang Qiquan, Fei Yuzhou, Ni Junwen. Model Study for Early Warning System of Urban Road Intersection Based on the Back Propagation Neural Network. American Journal of Embedded Systems and Applications. Vol. 4, No. 1, 2016, pp. 1-6. doi: 10.11648/j.ajesa.20160401.11

Received: August 22, 2016; Accepted: September 9, 2016; Published: October 9, 2016

\begin{abstract}
The urbanization is the sign of advanced development for an urban. In recent years, with the development of science, technology and economy and the rise of urban car ownership, urban road traffic became a severe problem. There occurred a huge number of urban road traffic accidents frequently. To study and find insufficiency for the research status at home and abroad, the four aspects --"man - vehicle - road - environment" are analyzed, and the comprehensive analysis of the present safety situation of urban road intersection is made. Selecting one in seven important influencing factors of urban road intersection index as a Back Propagation (BP) neural network input, the early warning model, based on BP neural network, is established. Data of existing urban road intersections is analyzed, and the results show that the BP neural network can be well applied to early warning and forecast model analysis of urban road intersection accident, thus it facilitates for the traffic administrative department of the city road intersection to predict the accident frequency of urban road intersection for the traffic accident in the future, take appropriate intervention measures and improve the safety status of urban road intersection.
\end{abstract}

Keywords: Urban Road Intersection, Warning System, BP Neural Network

\section{Introduction}

With the development of cities and the improvement of science and technology, traffic safety has become a hot topic in modern society. City road widening and the sharp increasing with private cars all reflect the increasing traffic demand. Travelling demand increase at the same time, however. The lack of driving experience, large traffic flow and traffic order have brought great hidden trouble to traffic safety [1]. China is one of the most road traffic conflict happened places. According to statistics of the ministry of public security, in 2014, there were 196000 casualties, 58000 deaths and 211000 people's injuries in road intersection traffic conflicts in our country.

At present, our country still has some gap in early warning theory of systemic research with the international level in the field of traffic safety. Domestic scholars (Wang Qiquan, Feng Zhibin), according to the core of the basic theory of safety evaluation [2], with traffic accident statistics as the evaluation index, put forward the clustering analysis method. Jian-min Xiong [3] and others studied for the condition of the pavement under the special environment of temperature and humidity monitoring system in view of the snow disaster in southern China in 2008 and the road traffic paralysis. Liu Qing, Yu Kang of the Wuhan University of Technology in the context of the highway research on early warning management system, put forward the general design requirement for the freeway traffic early warning basic composition and the evaluation method of management system which play an important role in highway safety.

In this paper, starting from the influence factors of traffic safety, according to the intersection accidents statistics and data for the whole year in the Beijing 25 road intersections, the author selected road intersection index factors, use the BP neural network to establish model of urban road intersections safety early warning system and deepen the domestic research of early warning system for urban road intersections.

\section{City Road Intersection Safety Situation Analysis}

\subsection{Urban Road Intersection Safety Influence Factors Analysis}

Road intersection system is made up of four complex 
system aspects--"human, vehicle, road and environment", mutually influencing between system components and coordinating with each other. Any kind of traffic factors became unstable or unreliable could lead to chaos in the system, resulting in traffic safety accidents.

\subsection{Human Factors}

Human factors are mainly divided into motor vehicle drivers, a non-motor vehicle drivers and pedestrians. As the main participants in the road intersection system, more than $90 \%$ of the accidents are caused by the motor vehicle drivers. The motor vehicle drivers in the system are dynamic variable factors. Ages, driving experiences, gender, personality and other reasons can easily lead to traffic accidents. The immediate cause of most of the traffic accidents is the error of the drivers' observations, judgment and operations and so on, generally included as follows:

(1) Fatigue driving, carelessness of keeping safe distances between the cars;

(2) Good road condition, too fast speeding;

(3) Behaviors to save time: no accordance with the rules of the road driving, speeding, grabbing line in yellow-light, not slowing down no traffic lights command crossroads, etc.

(4) Drink driving, illegal overtaking, reverse, etc. according to driver's body, physiology, age, emotional factors, such as the cause of frequent occurrence of road traffic accidents.

A non-motor vehicle drivers, pedestrians and passengers in the road traffic system belong to the vulnerable groups, but the humans' unsafe behaviors are often the causes of road intersection accidents. The survey data show that, in the motor vehicle drivers and pedestrians in road traffic accidents with illegal behaviors, nearly seventy percent of the reasons are running red light, illegal encroachments and illegal into the way.

Our country still belongs to developing countries. Because of the lack of funds, related research and construction, China's current road traffic conditions and various traffic facilities are not complete, which cannot achieve the vast majority of motor vehicles, non-motor vehicles and pedestrians' traffic. There were a lot of people, vehicles and non-motor vehicles mixed traffic.

\subsection{Vehicle Factor}

The vehicle's performances in poor and mechanics function failures are the two main reasons of motor vehicle traffic accidents. Motor vehicle parts failure is resulted that the driver did not check the vehicle parts before driving a motor vehicle or use for a long time with regular motor vehicle maintenance, leading the motor vehicle parts failures during driving and the motor vehicle without controlling. [4]

Motor vehicle has bad performance, which mainly refers to the braking performance of the motor vehicle. Motor vehicle power performance and the running smoothness of conveyor chains are not in conformity with the relevant standards and regulations of motor vehicle detection, make the motor vehicles out of control in the process [5].

\subsection{Road Factor}

The main reason is the road of defects caused by traffic accidents. In urban road intersection, accidents caused by the reason of road accident quantity are less [6], and a few of the accident was due to the road, road uneven subsidence, rain or rain the road slippery ice [7].

\subsection{Environmental Factors}

The mainly divide into two further factors--road's surrounding environments and traffic management facilities.

Roadways surrounding environments include the surrounding vegetation, architecture, town, road lighting, weather, road surface materials, etc. In some road intersections built-up with shops, billboards, neon, view will appear a large number of blind areas, making the driver s' bad observations of road conditions or distractions affect their judgment, thus some sudden stimulation can make the drivers ignore the observation of the surrounding cause traffic accidents.

Traffic management facilities, commonly used traffic management facilities, have traffic signs, lights, guardrail and pavement marking facilities. Road signs are the most commonly used traffic management facilities including traffic signs, lights. For safety, an important role plays reducing the level of potential accidents. [8] [9]

\section{Building Early Warning System for Urban Road Intersection Model}

\subsection{The Research Methods}

The BP neural network is the most important artificial neural network, widely used by many scholars in different fields. BP neural network is made up of three layers of network--the input layer, hidden layer and output layer. Each level has many neurons linked, constituting with the mapping network. According to a large number of studies have shown that strong mapping function of the three-layer structure of the BP neural network, any complex nonlinear mapping functions can be implemented successfully.

\subsection{BP Neural Network in MATLAB Implementation}

BP neural network is mainly used in analysis; it is a model to specify animal's behavior characteristics, and an algorithm mathematical model for distributed parallel information processing. BP neural network depends on the complexity of the system, adjusting the internal relations among a large numbers of nodes connected, so as to achieve the purpose of processing information. [10]

Neural network toolbox contains many functions used for the design of BP neural network and the analysis. When we use the BP neural network to forecast, we mainly use the following functions:

(1) Newff functions - create a BP neural network [11]

The commonly used format of this function is shown 
follows:

$$
\text { net }=\text { newff([S1S2 ..S(N-1)], }\{\text { TF1TF2 ..TFN }\}, \mathrm{BTF})
$$

Above $\mathrm{PR}$ is a $\mathrm{R} * 2$ dimensional matrix in the type, consist of the maximum and minimum values of each group of input elements; $[\mathrm{S} 1 \mathrm{~S} 2 \ldots \mathrm{S}(\mathrm{N}-1)]$ is the umber of neurons in each layer; The elements in $\left\{\mathrm{TF}_{1} \mathrm{TF}_{2} \ldots \mathrm{TF}_{\mathrm{n}}\right\}$ the respectively adopted by the transfer function of each layer neurons; $B T F$ indicates the BP neural network training, training adopted by the function; Back propagation is the generated BP network object; newff, at the same time the generation of the BP neural network, automatically initialized weights and pavilion in every layer of the network value. The user can, according to their different needs, redefine the layers of network weights and the rich value of the initialization function.

(2) Network function training, a BP neural network is set up and, after the initialization, can do the network training, commonly used the syntax of the format as follows:

$$
\text { net=train(net, } \mathrm{P}, \mathrm{T})
$$

In this function, $\mathrm{P}, \mathrm{T}$ are the training sample matrix and the target output matrix respectively; net is the untrained network for function newff. Network training and function train call a function to create the training of the BP neural network in the initial setting function and the algorithm for neural network training. Once the initialization of the network weights and threshold it can be trained according to the network input and target output to complete the function approximation, pattern classification, prediction, etc. During the network training, the weights of BP neural network and rich value continuously improve the performance of the network function and reduce to a minimum. In this paper, the urban road intersection safety prediction and the selection of initial parameters are as follows:

Every step interval 50 shows training results, allowing the largest training steps into 5000 steps, taking learning rate 0.01 ;

(3) network simulation function Sim

Sim function is used for simulation of neural network, and it can be used to test samples for trained network inspection. At the same time, for a given network input vector P, network object net's the output layer results yare returned. Common calls format as follows:

$$
\mathrm{y}=\operatorname{sim}(\text { net, } P)
$$

\section{Instance Analysis}

\subsection{Early Warning System Index Selection}

First of all the factors affecting the safety of road intersection, the human factor is the biggest test of the entire system, but for pedestrians and drivers, driving behavior characteristics and psychological change process for security early warning model of real-time data analysis are more difficult to conduct quantitative test. To facilitate quantitative detection, this article selects the intersections of pedestrians' drivers as input parameters.

And poor performance of the vehicle and parts function failure of the two major factors belongs to the amount of uncontrollable factors in the early warning system. At the same time, comparing to other safety factors, reasons caused by the vehicle road intersection in total events are in a less important role, so it would not be selected in this system.

In the analysis of urban road intersection safety influence factors, the easiest and the most accurate quantifiable factors mainly includes the roads and traffic environment factors. In the part of road environment factors, this article selects the consists of traffic signs, traffic line markings and light way of intersection traffic to control effect coefficient to evaluate the intersection traffic signal control factors; Chosen by crossing intersection electronic monitoring equipment for annual average daily traffic, left the proportions of the vehicles, trucks, intersection traffic flow indicators such as total import lanes to as input parameters of the model. That is the construction of model parameters.

To sum up, this paper established the driver, pedestrian traffic controlling method effect coefficient, the annual average traffic, the proportions of the vehicles, trucks, intersection import lanes of total 7 indexes as the BP artificial neural network input parameters. Respectively is:

(1) X1-the pedestrian driver. According to the survey period, it is the number of pedestrian violation and the ratio of the total number of pedestrian crossing.

(2) X2-the intersection type influence coefficient. Referencing literature, probabilities of accident in different intersections differ, determining coefficient of different intersection: four branches in the intersection, cross is $1, \mathrm{X}$ is 1.35 ; Three branches in the intersection, the $\mathrm{T}$ crossing is $1.9, \mathrm{Y}$ crossing is 2.1 ;

(3) X3-the intersection traffic control factors. According to under different traffic control method, the probabilities of accident in the road intersection to distinguish the different intersection traffic control coefficient of influence: the intersection signal good level is 0.78 ; the only sign is 1.48 ; marking intersection with police commanding is 0.58 ; signal with sign and marking is 1 ; with lights and police is 0.73 ; without traffic control is 1.50 ;

(4) X4-the annual average daily traffic; possibly accurate to a season or a month, etc. the corresponding unified statistical time range of other variables;

(5) X5-the truck scale,

(6) X6-the left turn vehicles;

(7) X7-the total import lanes for intersection;

\subsection{Sample Data}

Through related literature material consulting, sorting out and analyzing, the author obtained the related indexes and the number of accident data in 25 typical urban road intersections. According to the above content of urban intersection safety situation analysis and early warning system index selection, the author will choose 25 Beijing urban road intersections in the related data as the research objects. The selection of the data of 21 crossing uses BP neural network model for prediction of accident frequency, and the rest four intersections as for prediction data of the BP neural network to 
predict the output layer. Finally, the model predicted results of the reliability and superiority and inferiority of evaluation model are evaluated and analyzed.
The following table 1 and table 2 are the number of accidents in 25 urban road intersections and the intersection factor value table.

Table 1. Sample data of each intersection accident frequency

\begin{tabular}{|c|c|c|c|c|c|}
\hline $\begin{array}{l}\text { Intersection } \\
\text { serial number }\end{array}$ & Intersection name & $\begin{array}{l}\text { accident } \\
\text { frequency }\end{array}$ & $\begin{array}{l}\text { Intersection } \\
\text { serial number }\end{array}$ & Intersection name & $\begin{array}{l}\text { accident } \\
\text { frequency }\end{array}$ \\
\hline 1 & Er Lizhuang crossing & 7 & 14 & Kai Yangli street 3 & 5 \\
\hline 2 & Xihuan road and Changrong street & 6 & 15 & South station west door crossing & 5 \\
\hline 3 & Yulin south road crossing & 3 & 16 & Ronghua road and Yuncheng street & 4 \\
\hline 4 & Long Xiang crossing & 5 & 17 & North dust town and xingsheng street & 5 \\
\hline 5 & Xingfu crossing and Xingchang crossing & 5 & 18 & Beihuan road crossroad & 7 \\
\hline 6 & Ronghua road and east horse road & 4 & 19 & Xihuan road and Wenhuayuan road & 7 \\
\hline 7 & Taiping bridge crossroad & 6 & 20 & Donghuan road and Rongjing street & 7 \\
\hline 8 & Kechuang street 5 crossing & 3 & 21 & Xinde street & 5 \\
\hline 9 & Xihuan road and tianbao north street & 7 & 22 & Xihuan road and Tongji street & 4 \\
\hline 10 & Ronghua road and Xingsheng street & 2 & 23 & Yongchang road and Kangding street & 4 \\
\hline 11 & Xihuan road and Yongchang road & 1 & 24 & Under Zhixin bridge & 5 \\
\hline 12 & Ronghua road and Jingxiu street & 2 & 25 & Ronghua road and Rongjing street & 4 \\
\hline 13 & Xinkang crossing & 4 & & & \\
\hline
\end{tabular}

Table 2. Intersection factors numerical tables.

\begin{tabular}{|c|c|c|c|c|c|c|c|}
\hline crossing & X1 & $\mathrm{X2}$ & X3 & X4 & X5 & X6 & $\mathbf{X 7}$ \\
\hline 1 & 0.2207 & 1.9 & 0.78 & 48096 & 0.1447 & 0.3725 & 15 \\
\hline 2 & 0.1248 & 1 & 1 & 44748 & 0.1698 & 0.2565 & 21 \\
\hline 3 & 0.0946 & 1 & 0.78 & 23148 & 0.1889 & 0.0877 & 16 \\
\hline 4 & 0.1066 & 1 & 1 & 36948 & 0.1494 & 0.2501 & 19 \\
\hline 5 & 0.6117 & 1 & 0.78 & 39312 & 0.2125 & 0.1768 & 20 \\
\hline 6 & 0.4891 & 1.9 & 0.78 & 32496 & 0.2178 & 0.4036 & 10 \\
\hline 7 & 0.3389 & 1 & 1 & 95412 & 0.2486 & 0.2404 & 27 \\
\hline 8 & 0.0001 & 1 & 1 & 47544 & 0.2754 & 0.1848 & 15 \\
\hline 9 & 0.2759 & 1 & 1 & 71028 & 0.3315 & 0.2354 & 13 \\
\hline 10 & 0.0001 & 1 & 0.78 & 29388 & 0.1601 & 0.1058 & 16 \\
\hline 11 & 0.2308 & 1.35 & 1 & 16344 & 0.4835 & 0.1696 & 12 \\
\hline 12 & 0.0001 & 1 & 1 & 26028 & 0.2069 & 0.0737 & 16 \\
\hline 13 & 0.4579 & 1 & 0.78 & 52704 & 0.1272 & 0.0525 & 13 \\
\hline 14 & 0.5385 & 1.9 & 1 & 48444 & 0.1022 & 0.088 & 10 \\
\hline 15 & 0.5149 & 1.9 & 1 & 50124 & 0.1037 & 0.1586 & 13 \\
\hline 16 & 0.3396 & 1.9 & 1 & 40512 & 0.1205 & 0.1263 & 9 \\
\hline 17 & 0.1385 & 1 & 0.78 & 50376 & 0.2528 & 0.1795 & 25 \\
\hline 18 & 0.0737 & 1 & 1 & 62208 & 0.3396 & 0.1657 & 16 \\
\hline 19 & 0.0192 & 1.9 & 0.78 & 67980 & 0.253 & 0.1805 & 15 \\
\hline 20 & 0.0397 & 1 & 1 & 47052 & 0.2604 & 0.1428 & 12 \\
\hline 21 & 0.2566 & 1 & 1 & 41160 & 0.3491 & 0.2102 & 19 \\
\hline 22 & 0.03257 & 1 & 0.78 & 31536 & 0.3516 & 0.2059 & 12 \\
\hline 23 & 0.02933 & 1 & 1 & 27696 & 0.3791 & 0.1637 & 16 \\
\hline 24 & 0.0324 & 1 & 0.78 & 45372 & 0.2533 & 0.1667 & 16 \\
\hline 25 & 0.0105 & 1 & 0.78 & 47436 & 0.1676 & 0.1369 & 20 \\
\hline
\end{tabular}

\subsection{Results and Error Analysis}

According to the prediction model by the 25 road intersection operation, we selected the data before 21 road intersection as BP neural network training samples, and the number of road traffic accidents samples will be prediction test samples of four intersections. Statistical analysis using MATLAB software, based on the BP neural network prediction model of sample training, the program runs after convergence diagram as shown in figure 1.

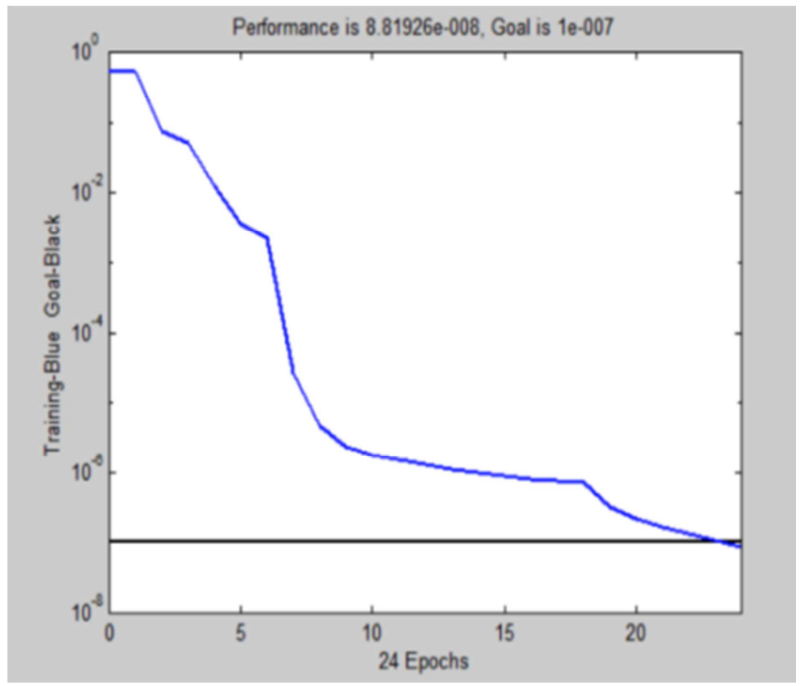

Figure 1. The BP neural network training curve

From figure 1, as we can see, using the BP neural network forecast the number of road intersection accident when the samples training model in the iteration after 24 times meeting the requirements of the error of the target. The training error is $8.81926 \mathrm{e}-008$, and the target error of 1e-007.Curve is gentle, and can produce relatively accurate prediction results. The error is small, not easy to generate large deviation in the sample prediction and able to accurately forecast. Then we reuse trained prediction model of urban road intersection accident prediction, prediction results and error analysis as shown in table 3 .

Table 3. Samples predicted results and error.

\begin{tabular}{llll}
\hline $\begin{array}{l}\text { Sample } \\
\text { number }\end{array}$ & $\begin{array}{l}\text { The actual number of } \\
\text { road accidents }\end{array}$ & $\begin{array}{l}\text { The BP neural network } \\
\text { predictive value }\end{array}$ & $\begin{array}{l}\text { Relative } \\
\text { error (\%) }\end{array}$ \\
\hline 22 & 4 & 4.3149 & $7.87 \%$ \\
23 & 4 & 4.3847 & $9.62 \%$ \\
24 & 5 & 5.2168 & $4.34 \%$ \\
25 & 4 & 4.1387 & $3.47 \%$ \\
\hline
\end{tabular}


According to table 3, predictive value of BP neural network is close to the actual value. The error is less than 0.4000 , and the relative error less than $10.00 \%$. Relatively comparing with samples 22 and 23, samples 24 and 25 have small errors which values are nearly as twice, leading to certain volatility the sample prediction results under the condition of different samples. Therefore, based on sample data of road intersection simulation training model of former 21 samples, BP neural network method can predict the number of the rest four road intersection traffic accidents, and the error range is small. When reliable road traffic accident samples are equipped, the BP neural network prediction deviation is small. Prediction results can be applied in urban road intersection safety forecast. But, for the different intersection, BP neural network prediction accuracy and relative error are different. For the bigger prediction error, the existence impacts of the actual forecast are needed to be considered, in order to get more accurate forecasts through certain measures to eliminate these factors. By using the BP neural network prediction, early warning model of urban road intersections can be established against the possibility of traffic accidents with preventions in advance and allocation of necessary emergency rescue facilities, it can reduce the traffic accident rates and the traffic accident damage.

\section{Conclusion}

Early warning system for urban road intersections is the ultimate goal of research about the future of scientific prediction of accident frequency in road intersection, and according to the historical data and predicted data, it evaluate the security status of the current road intersection. For the government or the traffic administrative department of personnel, it takes corresponding measures to reduce the incidence of road intersection and enhance security. Based on the general situation of urban road intersection and security influence factors analysis to set up index system of early warning system for road intersection, finally it uses BP neural network to build the early warning model in urban road intersection. Research conclusions are as follows:

(1) According to the statistical information, there is a detailed comprehensive analysis of the statistical data by "person, vehicle, road and environment" of the system in general situation and the factors influencing the safety in road intersection.

(2) In urban road intersection, with comprehension of safety situation, we choose the selection --pedestrians' driver, traffic controlling effect coefficient, the annual average traffic, and the left proportions of the vehicles, trucks, and intersection import lanes of total 7 indexes as the input parameter prediction model for urban road intersection construction of early warning system model.

(3) Choosing the BP neural network to establish early warning system with Beijing's 25 road intersection data, using statistical analysis software mat lab to training sample data and going through 24 iterations, finally we could got the forecast data. Predicted results show and prove that BP neural network can be well applied to urban road intersection safety forecast, but the BP neural network awfully predict a certain amount of error.

Urban road intersection is a complex dynamic system. There are multiple components of dynamic factors. By the author's level, it will be limited to the qualitative or quantitative analysis and the paper is still some deficiency:

(1) Early warning index system of urban road intersection choice. In the system with the above analysis in the road intersection system, because human factors caused by accidents accounted for the total number of factors in the road intersection system quantitative analysis, the current monitoring content can't identify the persons' ages, driving experiences, and even genders. So the author has chosen relatively easy to obtain the intersections data as input parameters of the model, thus the precision of prediction and actually will appear certain differences.

(2) The choice of method model. This paper chose the BP neural network model building, the article has proved that the BP accident can be well applied to urban road intersections and it is the number of prediction, but it also has certain limitation of BP neural network that BP neural network cannot intuitive to predict the dynamic changes of the various input parameters, and also cannot predict the change of composition, so the early warning system in urban road intersections can complete a little function.

\section{Acknowledgment}

This paper is supported by general educational improving project of China Institute of Industrial Relations (JG1529).

\section{References}

[1] Road traffic accident statistical yearbook of the People's Republic of China (2012). The ministry of public security traffic management bureau. 2013.5.

[2] Analysis and research on road traffic safety evaluation [A]. Wang Qiquan, Feng Zhibin. China association of occupational safety and health. The Chinese association of occupational safety and health Years academic essays in 2007[C]. Occupational safety and health association: China, 2007-6.

[3] The embankment settlement experiment research and numerical simulation [J]. Jian-min, Xiong Shengji, Yu Qintian. Journal of Huazhong University of science and technology (city science edition), 2008, 2008:54-56.

[4] The establishment of the highway traffic safety early warning management system (English) [J]. Liu Qing, Wu Yanzi. Journal of Wuhan university of technology (transportation science and engineering), 01 2003:2003-424.

[5] Road traffic safety management planning [M]. Yan Baojie, Zhang Shengrui. Beijing: China railway mountain edition du, 2008.06. 1-3. 
[6] The world health organization, the World Bank. World road traffic injuries report [R] (2004), 2004.

[7] The road traffic safety and the back reflection technology [M]. Liujian Jun Beijing: people's traffic mountain edition du, 2009. 19-57.

[8] Road traffic safety management in our country main problem analysis and countermeasures study [J]. Zhou Xin, Wanshou en.The safety production science and technology of China. 2007. 3 .
[9] Introduction to the road traffic safety [M]. Zheng Anwen, Yuan Hongwei. Beijing: mechanical industry mountain edition. 2010, 3-40.

[10] Based on the application of BP neural network [J], Wang Linlin, Anyang normal college physics and electrical engineering college, Henan, Anyang, 2014, 1.

[11] Analysis and improving way of BP ANN in predicting time series data [J]. WANG Wei, ZHANG Yingtang, Computer Engineering and Design, 2007, 28(21). 